\title{
Prolonged pituitary down-regulation with full-dose of gonadotropin-releasing hormoneagonist in different menstrual cycles: a retrospective cohort study
}

\author{
Yingfen Ying ${ }^{1}$, Tanchu Yang ${ }^{1}$, Huina Zhang ${ }^{1}$, Chang Liu ${ }^{1}$, Junzhao Zhao ${ }^{\text {Corresp. } 1}$ \\ ${ }^{1}$ Reproductive Center, Department of OB \& GYN, The Second Affiliated Hospital of Wenzhou Medical University, Wenzhou, Zhejiang, China \\ Corresponding Author: Junzhao Zhao \\ Email address: joyce08@126.com
}

Background: The efficiency of prolonged down-regulation caused by a full-dose of gonadotropin-releasing hormone agonist ( $\mathrm{GnRH}-\mathrm{a}$ ) injected during different menstrual phases has not yet been researched. Our goal was to evaluate the effects of GnRH-a, which was used in different phases of the menstrual cycle in patients undergoing in vitro fertilization and embryo transfer (IVF-ET). Methods: This was a retrospective cohort study. Three hundred and twenty patients received a prolonged pituitary down-regulated fulldose $(3.75 \mathrm{mg})$ of triptorelin in the early follicular phase, and 160 patients received the same full-dose of triptorelin during the mid-luteal phase. Clinical and laboratory outcomes were compared between the two groups. Results: The basic characteristics of the two groups were comparable. The mean number of retrieved oocytes, fertilized oocytes, cleavage oocytes and good quality embryos were comparable between the two groups. Although there was a higher antral follicle count (AFC), cyst formation rate, fertilization rate and cleavage rate in the follicular phase group, no statistically significant effects were seen on implantation rate $(41.15 \%$ vs. $45.91 \%)$, clinical pregnancy rate $(60.38 \%$ vs. $61.36 \%)$, ongoing pregnancy rate $(57.74 \%$ vs. $57.58 \%)$, live birth rate $(56.23 \%$ vs. $57.58 \%)$ or early abortion rate ( $2.64 \%$ vs. $3.79 \%)$ per fresh transfer cycle. Moreover, severe ovarian hyper-stimulation syndrome (OHSS) rates at the early stage (1.89\% vs. $2.27 \%)$ were low in both groups. Conclusions: Prolonged pituitary down-regulation achieved by utilizing a full-dose of GnRH-a administrated in either phase of the menstrual cycle can have a positive effect on ongoing pregnancy rate and live-birth rate per fresh embryo transfer cycle. Ovarian cyst formation rate was higher in the follicular phase group, but this did not have any adverse impact on clinical results. 
1 Prolonged pituitary down-regulation with full-dose of gonadotropin-releasing hormone

2

3

4

5

6

7

$8 \quad$ Yingfen Ying ( the first author)

$9 \quad$ Email:20918182@zju.edu.cn

10 Affiliated institution: Reproductive Center, department of OB \& GYN, the

11 Second Affiliated Hospital of Wenzhou Medical University, Wenzhou, Zhejiang, China

12 Tanchu Yang

13 Email:815034046@qq.com

14 Affiliated institution: Reproductive Center, department of OB \& GYN, the

15 Second Affiliated Hospital of Wenzhou Medical University, Wenzhou, Zhejiang, China

16 Huina Zhang

17 Email:Huina.zh201@163.com

18 Affiliated institution: Reproductive Center, department of OB \& GYN, the 
19 Second Affiliated Hospital of Wenzhou Medical University, Wenzhou, Zhejiang, China

20 Chang Liu

21 Email:632027539@qq.com

22 Affiliated institution: Reproductive Center, department of OB \& GYN, the

23 Second Affiliated Hospital of Wenzhou Medical University, Wenzhou, Zhejiang, China

24 Junzhao Zhao (Corresponding author)

25 Email:joyce08@126.com

26 Affiliated institution: Reproductive Center, department of OB \& GYN, the

27 Second Affiliated Hospital of Wenzhou Medical University, Wenzhou, Zhejiang, China

30 ABSTRACT

31 Background: The efficiency of prolonged down-regulation caused by a full-dose of 32 gonadotropin-releasing hormone agonist (GnRH-a) injected during different menstrual phases

33 has not yet been researched. Our goal was to evaluate the effects of GnRH-a, which was used in 34 different phases of the menstrual cycle in patients undergoing in vitro fertilization and embryo 35 transfer (IVF-ET).

36 Methods: This was a retrospective cohort study. Three hundred and twenty patients received a

37 prolonged pituitary down-regulated full-dose $(3.75 \mathrm{mg})$ of triptorelin in the early follicular phase, 
38 and 160 patients received the same full-dose of triptorelin during the mid-luteal phase. Clinical

39 and laboratory outcomes were compared between the two groups.

40 Results: The basic characteristics of the two groups were comparable. The mean number of 41 retrieved oocytes, fertilized oocytes, cleavage oocytes and good quality embryos were 42 comparable between the two groups. Although there was a higher antral follicle count (AFC), 43 cyst formation rate, fertilization rate and cleavage rate in the follicular phase group, no 44 statistically significant effects were seen on implantation rate (41.15\% vs. $45.91 \%)$, clinical 45 pregnancy rate $(60.38 \%$ vs. $61.36 \%)$, ongoing pregnancy rate $(57.74 \%$ vs. $57.58 \%)$, live birth 46 rate $(56.23 \%$ vs. $57.58 \%)$ or early abortion rate $(2.64 \%$ vs. $3.79 \%)$ per fresh transfer cycle.

47 Moreover, severe ovarian hyper-stimulation syndrome (OHSS) rates at the early stage (1.89\% vs. $48 \quad 2.27 \%$ ) were low in both groups.

49 Conclusions: Prolonged pituitary down-regulation achieved by utilizing a full-dose of GnRH-a

50 administrated in either phase of the menstrual cycle can have a positive effect on ongoing 51 pregnancy rate and live-birth rate per fresh embryo transfer cycle. Ovarian cyst formation rate 52 was higher in the follicular phase group, but this did not have any adverse impact on clinical 53 results.

\section{INTRODUCTION}

55 Over the last three decades, gonadotropin-releasing hormone agonists (GnRH-a) were the most 56 commonly used drugs for controlled ovarian hyperstimulation $(\mathrm{COH})$ in assisted reproductive 
57 procedures. These drugs provided many benefits, such as the recovery of a larger number of 58 oocytes, prevention of premature luteinizing hormone (LH) surge, luteinization, and a lower 59 cycle cancellation rate[Haydardedeoğlu \& Kılıçdağ, 2016; Siristatidis et al., 2015; Xiao, Su 60 \& Zeng, 2014]. Utilizing GnRH-a has been considered the gold standard for COH. Different dosages and formulations of GnRH-a were devised. It has been noted that clinical pregnancy rate might increase when patients are given a period of artificial amenorrhea caused by GnRH-a [Cai, et al., 2018; Liao et al., 2015; Martínez et al., 2014; Ren et al., 2014]. One full-dose depot of long-acting GnRH-a per $\mathrm{COH}$ cycle would be more convenient for women than short-acting GnRH-a injections because there are fewer injections, leading to greater compliance as well as fewer incidences of infection [Cheon et al., 2008; Gao et al., 2014]. Liao et al. reported that clinical pregnancy rates in patients who received a depot GnRHa regimen was higher than that of the daily low-dose group (57.5\% vs. 46.9\%) [Liao et al., 2015]. The prolonged regimen can be divided into two different types, according to the time that the GnRH agonist is administered. The first type, the follicular phase, is where the GnRH agonist is

71 injected between the first and third days of the menstrual cycle; the second type, the mid-luteal 72 phase, is where the drug is administered in the middle of the previous luteal cycle. It was 73 concluded by Broekmans et al. that a single administration of GnRH-a either in the mid-luteal or 74 the early follicular phase reached a high degree of similarity in pharmacodynamics response, which rapidly induced a reversible hypogonadotropic and hypogonadal condition. Pituitary and ovarian suppression was maintained until the beginning of the 8th week [Broekmans, et al., 
77 1992].

78 Until now, the clinical efficiency of prolonged down-regulation caused by a full-dose of GnRH-a

79 injected during different menstrual phases has not yet been researched either at home nor abroad.

80 So, does prolonged down-regulation caused by a full-dose of GnRH-a injected during different

81 menstrual phases cause a similar high pregnancy rate? The aim of this study was to compare the

82 clinical efficacy of a full-dose of the GnRH-a drug triptorelin in follicular and mid-luteal

83 regimens before $\mathrm{COH}$ through ongoing pregnancy and live birth, which are vital indicators in

84 suitable infertile couples. We can then explore whether one or both meet the clinical

85 requirements, and which one is better.

86 MATERIALS AND METHODS

\section{Study participants}

88 This was a retrospective study approved by the ethics committee of the Second Affiliated

89 Hospital of Wenzhou Medical University (Ethic Reference No: L-2018-19). This study was

90 performed on fresh IVF/ICSI ET cycles with $3.75 \mathrm{mg}$ triptorelin for prolonged pituitary down-

91 regulation injected in the follicular phase (Group 1) or mid-luteal phase (Group 2) of the

92 menstrual cycle from June 2016 to May 2017. Inclusion criteria were: (1) all patients were 20-38

93 years old with infertility due to: salpingemphraxis, endometriosis, polycystic ovarian syndrome,

94 polycystic ovary, male factors, or idiopathic causes; (2) a body mass index (BMI) $<28 \mathrm{~kg} / \mathrm{m}^{2}$; (3)

95 basal serum follicle stimulating hormone $(\mathrm{FSH})<12 \mathrm{IU} / \mathrm{L}$ and estradiol $\left(\mathrm{E}_{2}\right)<80 \mathrm{pg} / \mathrm{ml}$ were 
96

97

112 Ferring, Germany) during the follicular phase of the menstrual cycle in Group 1, in which

determined on the third day of the cycle. Uterine abnormalities such as müllerian malformations, fibroids, and adenomyosis were excluded. Patients that had received any ovulation-induction treatment within three months of study entry were also excluded. Different treatment regimens were performed simultaneously for the two groups of patients. Before a cycle was initiated, candidate patients were fully informed with detailed information of both protocols, including the time-point and duration of down-regulation, the pregnancy rate, and the potential risk of strong pituitary depression. Based on this information, patients made the decision to get prolonged pituitary down-regulation in the early follicular phase or in the mid-luteal phase. All the patients enrolled gave written informed consent for the whole procedure.

Of the 480 cycles that met the study criteria, 25 cycles in Group 1 and seven cycles in Group 2 were canceled because fewer than one oocyte was retrieved, and subsequently there were no available embryos. An additional 30 cycles in Group 1 and 21 cycles in Group 2 were canceled because a freeze-all of the embryos was performed. Therefore, 265 cycles of Group 1 and 132 cycles of Group 2 were observed (Figure 1).

\section{COH and IVF/ICSI procedure}

Pituitary desensitization was achieved with a single full-dose injection of $3.75 \mathrm{mg}$ (Triptorelin, ovarian stimulation would occur 32-38 days later. In Group 2, pituitary down-regulation was started in the mid-luteal phase with a full dose of $3.75 \mathrm{mg}$ triptorelin, and $\mathrm{COH}$ with 
gonadotropin would commence from 32 to 38 days after the single GnRH-a injection.

116 Successful pituitary down-regulation was confirmed after finding no antral follicles larger than

$1178 \mathrm{~mm}, \mathrm{E}_{2}$ concentration less than $50 \mathrm{pg} / \mathrm{ml}$, serum LH levels less than $5 \mathrm{IU} / \mathrm{L}$, and endometrium

118 thickness less than 5mm. COH with recombinant FSH (r-FSH) (Gonal-F, Merck Serono,

119 Switzerland) was started at one to four ampules (75-300 IU) and the dosage was adjusted

120 according to patients' BMI, antral follicular count (AFC), basal FSH level, and follicular growth

121 response. Transvaginal ultrasound and serum $\mathrm{E}_{2}, \mathrm{LH}$, and $\mathrm{P}$ levels were used to monitor ovarian

122 response. Recombinant LH (r-LH) (recombinant-LH, Merck Serono, Switzerland) was added at

123 the late stage of follicular growth when the LH level was $<0.5 \mathrm{IU} / \mathrm{L}$ with the dosage of $75 \mathrm{IU} \mathrm{r}-\mathrm{LH}$.

124 One dose of 5000-10000 IU human chorionic gonadotropin (hCG, Livzon, Guangdong, China)

125 was given when at least two follicles reached $18 \mathrm{~mm}$ in mean diameter under ultrasonograph.

126 Oocytes were retrieved $34 \sim 36$ hours later via vaginal ultrasound.

127 Oocytes were fertilized by IVF or ICSI $4 \sim 6$ hours after oocyte retrieval. Embryos were

128 incubated at $37^{\circ} \mathrm{C}$ under humidified gas phase of a mixture of $6 \% \mathrm{CO} 2,4 \% \mathrm{O} 2$ and $90 \% \mathrm{~N} 2$.

129 Embryo score was performed as described [Alpha Scientists in Reproductive Medicine and

130 ESHRE Special Interest Group of Embryology, 2011]. Normal fertilization was confirmed by the

131 presence of two pronuclear and two polar bodies $16 \sim 18$ hours post-insemination. Cleavage-

132 stage embryo score was based on the number of blastomeres, blastomere size, and the proportion

133 of fragments. Morulae-stage embryos were assessed on the basis of the proportion of compaction.

134 Blastocyst scoring was consisted of the expansion stage of blastocyst cavity expansion, density, 
135

136

137

138

139

140

141

142

143

144

145

146

147

148

149

150

151

152

153

154

cell number of inner cell mass, and the cohesion as well as regularity of trophectoderm [Alpha

Scientists in Reproductive Medicine and ESHRE Special Interest Group of Embryology, 2011].

Embryo transfer was determined according to the embryo quality, the state of endometrium and the physician's convenience. Under the guidance of abdominal ultrasound, embryo transfer was performed with at least one good quality embryo at the specific developmental stage. Luteal phase support was sustained with micronized progesterone (Utrogestan, Capsugel, Besins Manufacturing Beigium), $200 \mathrm{mg}$ orally administrated per day, and progesterone vaginal gel prolonged release (Crinone, Merck Serono, Switzerland), $90 \mathrm{mg}$ each day from the day of oocyte retrieval. Procedures of Group 1 and Group 2 were shown in Figure 2 and Figure 3 respectively. Serum $\beta$-hCG determination was performed 14 days after cleavage-stage embryo transfer, or 12 days after blastocyst transfer. Clinical pregnancy was defined as a transvaginal ultrasound detection of gestational sac(s) with pulsating heart beats four to five weeks after embryo transfer. Implantation rate was calculated as the ratio of the number of gestational sac(s) over the number of transferred fresh embryos. Early abortion was defined as a clinical pregnancy failing to reach the 12th gestational week. Ongoing pregnancy was defined as a clinical pregnancy that reached more than 12 gestational weeks.

\section{Statistical analysis}

The demographic data of the infertile patients, serum hormonal levels, stimulation requirements, and clinical outcome variables were all compared between the two groups. Analysis was performed with SPSS (version 22.0; IBM). Independent samples $t$-test or Mann-Whitney test 
155

156

157 158 159

160

161

162

163

164

165

166

167

168 169

were used for continuous variables. Dichotomous variables were analyzed by chi-square test or Fisher's exact test as required. Multivariable logistic regression analysis was used to test the association between the selected variables and the probability of live-birth rates. In all analysis, $P$ $<0.05$ was considered statistically significant.

\section{RESULTS}

The general characteristics of the patients of both groups were comparable. The average age ( $30.58 \pm 3.57$ vs. $31.14 \pm 4.18$ yrs) and the mean duration of infertility ( $3.58 \pm 2.44$ vs. $3.80 \pm 2.62$ yrs) were similar in both groups. There were no significant differences in the body mass index (BMI:21.28 \pm 2.56 vs. $21.79 \pm 2.65$ ) nor the proportion of primary infertility between the two groups. The basal FSH level of Group 1 was less than that of Group 2, though without significant difference between the patient cohorts (Table 1). There was more AFC on the day $\mathrm{COH}$ commenced in Group 1 ( $14.68 \pm 5.57$ vs. $12.61 \pm 5.64, P=0.001$; Table 1). Considering the basal FSH level and AFC, the ovarian reserve function of Group 1 was better than that of Group 2. The average serum $\mathrm{LH}, \mathrm{E}_{2}$, and $\mathrm{P}$ levels were similar in both groups $32-38$ days after pituitary desensitization. The serum LH, P, $\mathrm{E}_{2}$ levels and endometrial thickness were equivalent on the day of hCG in both groups. The duration of ovarian simulation and the total dosage of ovarian stimulating drug were similar in both treatment protocols (Table 1).

The number of oocytes retrieved, fertilized, and cleaved, and the number of good quality embryos in both procedures were similar. However, the ratio of fertilization and cleavage was 
174 significantly different between treatment groups $(68.81 \%(2116 / 3075)$ vs. $65.67 \%(1058 / 1611)$

$175 P=0.03),(97.26 \%(2058 / 2116)$ vs. 94.61\%(1001/1058) $P<0.001)$ (Table 2). The rate of ovarian

176 cyst formation was higher in Group $1(6.42 \%(17 / 265)$ vs. $0 \%(0 / 132) P=0.003)$ (Table 2$)$.

177 Clinical pregnancy, embryo transfer, ongoing pregnancy, early abortion, and live birth rates were 178 comparable between treatment protocols. The incidence of severe ovarian hyperstimulation 179 syndrome (OHSS) at an early stage was not significantly different between groups. The 180 proportion of freezing all embryos was equivalent in both groups, 10.17 vs. 13.73 percent (Table $1812)$.

182 We divided Group 1 into two subgroups, with and without cyst development, and compared their 183 laboratory and clinical outcomes. The proportion of good quality embryos, clinical pregnancy 184 rate, ongoing pregnancy rates, early abortion rate, and live birth rate were all higher in the cyst 185 subgroup, but the differences were not statistically significant (Table 3). The average $\mathrm{E}_{2}$ level on 186 the starting day in the cyst subgroup $(28.71 \pm 11.20 \mathrm{pg} / \mathrm{ml})$ was more comparable than that in the 187 non-cyst subgroup. Only one patient's $\mathrm{E}_{2}$ level on the starting day was more than 50pg/ml not 188 shown in Table 3. Initial and total doses of FSH were higher in the cyst subgroup, but there was 189 no statistical difference (Table 3).

190 To evaluate the contribution of different time-points of down-regulation on the difference in live191 birth rate, a multivariate logistic regression model was used to analyze some related confounding 192 factors. The variables were basal FSH levels, AFC, P, LH and $\mathrm{E}_{2}$ levels on the commencing day 193 of stimulation, and endometrial thickness on the day of hCG. Moreover, other factors (age, BMI, 
194 progesterone elevation on the hCG day, number of good quality embryos on day 3) were also 195 selected based on clinical experiences, though they were similar in both cohorts. The Hosmer-

196 Lemshow goodness-of-fit chi-square test statistic was $4.099(P=0.848)$, which suggested that the 197 multivariable model was a good fit $(P>0.05)$. Adjusted by the previously mentioned potential

198 199

200

201

202

203

204

205

206

207

208

209

210

211

212

213

confounding factors, the odds ratio of prolonged down-regulation in the early follicular phase versus mid-luteal phase on the live-birth rate was 1.040 (95\% confidence interval, $0.997-1.084)$.

\section{DISCUSSION}

As expected and suggested by some researchers, a long-acting protocol with a full-dose of

GnRH-a caused pituitary side effects, inhibited ovarian steroidogenesis, and affected the differentiation of granulosa cells [Dor et al., 2000; Metallinou et al., 2012]. One review [Albuquerque et al., 2013] indicated that using a long acting GnRH-a for pituitary downregulation required more gonadotropins and a higher duration of ovarian stimulation. However, our data showed that the total dosage of gonadotropins in our study was lower in Group 1, 2243 IU versus 2440 IU in Group 2, both of which were slightly lower or similar to that of one-third or one-half dose of the GnRH-a depot published by certain studies [Chen et al., 2016]. That means a full-dose of GnRH agonist would not drastically increase the economic burden for patients. In addition, the effective duration of gonadotropins with respect to ovarian stimulation was 11.51 days for Group 1 versus 12.41 days for Group 2, which was similar to the duration of half-dose of GnRH agonist in long-acting regimens [Ren et al., 2013]. Moreover, the clinical and ongoing pregnancy rates achieved in this study were higher than those of procedures involving one-half 
214 dose or one-third dose of GnRH-a [Chen et al., 2016].

215 Optimal serum LH concentrations at the beginning of ovarian stimulation after pituitary down-

216 regulation with $\mathrm{GnRH}$ agonist is neither $<0.1 \mathrm{IU} / \mathrm{L}$ nor $>1 \mathrm{IU} / \mathrm{L}$, which are the levels of $\mathrm{LH}$

217 required in follicular development [Shahrokh Tehraninejad et al., 2017; Mochtar et al., 2017].

218 Excessive pituitary suppression, meaning less than $0.1 \mathrm{IU} / \mathrm{L}$ serum LH levels, requires

219 exogenous LH to increase the number of mature oocytes and good quality embryos [Razi et al.,

220 2014; Shahrokh Tehraninejad et al., 2017]. In contrast, insufficient pituitary suppression,

221 meaning more than $1 \mathrm{IU} / \mathrm{L}$ of serum LH concentration, has been proven to have adverse impacts

222 on oocyte and embryo quality and reduces embryo implantation potential and clinical pregnancy

223 rates [Loumaye et al., 1989]. Our data showed that serum LH levels at $32 \sim 38$ days of pituitary

224 suppression were 0.46 to $0.49 \mathrm{IU} / \mathrm{L}$, appropriate $\mathrm{LH}$ concentrations for starting $\mathrm{COH}$.

225 Our data simultaneously showed that serum LH concentrations on the day of hCG injection were

226 low in patients in both procedures, which was similar to the study of Gen-Hong Mao [Mao et al.,

227 2014], indicating positive endometrial receptivity. However, little is known about the effects of

$228 \mathrm{LH}$ on the endometrium regarding activation in IVF/ICSI cycles [Londra et al., 2016]. The

229 corresponding risk of severe early OHSS after using a long acting GnRH-a protocol was $1 \%$ to

$2306 \%$, similarly found in our research [Albuquerque, et al., 2013].

231 One of the detrimental effects of using GnRH-a is the formation of ovarian cysts, which may be

232 either functional or non-functional. In this research, prolonged pituitary down-regulation 
233 throughout the early follicular phase was shown to create many more ovarian cysts, most of

234 which were non-functional. The pathophysiological mechanism of cyst formation is unknown so

235 far. However, possible mechanisms have been published [Feldberg et al., 1989; Pereira et al.,

236 2016; McDonnell et al., 2014]. It is easy for ovarian cysts to form when initiated at the follicular

237 phase, especially in women with higher basal FSH levels during their menstrual cycle. Some

238 other explanations could be as follows: the effect of primary flare-up caused by the effect of

239 GnRH-a on gonadotropins; inadequate suppression of circulated gonadotropins following

240 hypophyzectomy; and the direct effect of $\mathrm{GnRH}$ agonists on ovaries and subsequent

241 steroidogenesis [Firouzabadi, Sekhavat \& Javedani, 2010]. Similarly, in this research, ovarian

242 cyst formation rate was higher in the group in which GnRH-a was administrated in the early

243 follicular phase. While some studies proposed that ovarian cyst formation not only compromised

244 the quality of oocytes and embryos but also increased cycle cancellation rates and decreased

245 pregnancy rates, these results were contrary to our findings [Qublan et al., 2006].

246 With one exception, all cysts were nonfunctional. We did not treat non-functional cysts, although

247 treatments such as draining the cyst via ultrasound-guided cyst puncture are available. Though

248 the initial dose and total dose of samples were much higher for the non-cyst subgroup, the rates

249 of good quality embryos, clinical pregnancy rate, continued pregnancy rates, and live birth rate

250 were all slightly but not significantly higher in the cyst subgroup. These results were consistent

251 with some studies in the literature [Firouzabadi, Sekhavat \& Javedani, 2010; Qublan et al., 2006].

252 Most participants in a survey of worldwide IVF centers believe that the presence of non- 
253 functional ovarian cysts does not influence the outcome and will therefore start stimulation,

254 while $28 \%$ of centers will go forward with the cycle only after aspirating this cyst [Tur Kaspa \&

255 Fauser, 2013].

256 Therefore, we believe that the non-functional ovarian cysts formed after GnRH agonist do not

257 affect the clinical outcomes. The effect of functional ovarian cysts on clinical outcomes after

$258 \mathrm{GnRH}-\mathrm{a}$ usage is controversial. There is insufficient evidence to determine whether drainage of

259 functional ovarian cysts prior to controlled ovarian hyperstimulation negatively influenced live

260 birth rate, clinical pregnancy rate, number of follicles recruited, or oocytes collected in women

261 with a functional ovarian cyst [McDonnell, Marjoribanks \& Hart, 2014]. In this research, the

262 formation rate of functional ovarian cysts was much lower than that of nonfunctional cysts.

\section{CONCLUSION}

264 In conclusion, our data suggested that prolonged pituitary down-regulation in the early follicular

265 phase can be as equally effective as that in the mid-luteal phase in fresh IVF/ICSI-ET cycles.

266 Ovarian cyst formation rate was higher in the follicular phase group, while this group still

267 showed comparable efficiency in the results of IVF performance and clinical outcomes.

268 Additional well-designed randomized trials that compare long acting GnRH agonists injected in

269 the follicular phase and in the mid-luteal phase are expected to demonstrate good IVF/ICSI

270 outcomes. 
272

273

274

275

276

277

278

279

280

281

282

283

284

285

286

287

288

\section{REFERENCES}

Albuquerque LE, Tso LO, Saconato H, Albuquerque MC, Macedo CR. 2013. Depot versus

daily administration of gonadotrophin-releasing hormone agonist protocols for pituitary down

regulation in assisted reproduction cycles. Cochrane Database Syst Rev 31(1):CD002808 DOI:

10.1002/14651858.CD002808.pub3.

Broekmans FJ, Bernardus RE, Berkhout G, Schoemaker J. 1992. Pituitary and ovarian suppression after early follicular and mid-luteal administration of a LHRHagonist in a depot formulation: decapeptyl CR. Gynecol Endocrinol 6(3):153-161.

Cai J, Liu L, Zheng J, Zhang L, Jiang X, Li P, Sha A, Ren J. 2018. Differential response of AMH to GnRH agonist among individuals: the effect on ovarian stimulation outcomes. $J$ Assist Reprod Genet 35(3):467-473.

Chen X, Feng SX, Guo PP, He YX, Liu YD, Ye DS, Chen SL. 2016. Does lower dose of longacting triptorelin maintain pituitary suppression and produce good live birth rate in long down-regulation protocol for in-vitro fertilization? J Huazhong Univ Sci Technolog Med Sci

36(2):215-220 DOI: 10.1007/s11596-016-1569-8.

Cheon KW, Song SJ, Choi BC, Lee SC, Lee HB, Yu SY, Yoo KJ. 2008. Comparison of clinical efficacy between a single administration of long-acting gonadotrophin-releasing 
289 hormone agonist (GnRHa) and daily administrations of short-acting GnRHa in in

290 vitrofertilization-

291 embryo transfer cycles. J Korean Med Sci 23(4):662-666 DOI: 10.3346/jkms.2008.23.4.662.

292 Dor J, Bider D, Shulman A, Levron J, Shine S, Mashiach S, Rabinovici J. 2000. Effects of

293 gonadotrophin-releasing hormone agonists on human ovarian steroid secretion in vivo and in

294 vitro results of a prospective, randomized in-vitro fertilization study. HumReprod 15(6):1225-

2951230.

296

297

298

299

300

301

302

303

304

305

Feldberg D, Ashkenazi J, Dicker D, Yeshaya A, Goldman GA, Dicker D, Goldman JA. 1989.

Ovarian cyst formation: a complication of gonadotropin-relasing hormone agonist therapy. Fertil Steril 51(1):42-44.

Firouzabadi RD, Sekhavat L, Javedani M. 2010. The effect of ovarian cyst aspiration on IVF treatment with GnRH. Arch Gynecol Obstet 281(3):545-549 DOI: 10.1007/s00404-0091195-9.

Gao J, Xu YW, Miao BY, Zhou CQ. 2014. Self-control study on reduced-dose depot versus daily administration of gonadotrophin-releasing hormone agonists for pituitary desensitization in in vitro fertilization cycles. J Obstet Gynaecol Res 40(7): 1901-1906 DOI: 10.1111/jog.12417. 
306 Haydardedeoğlu B, Kılıçdă EB. 2016. A novel approach using a minimal number of 307 injections

308 during the IVF/ICSI cycle: Luteal half-dose depot GnRH agonist following corifollitropin alfa

309

310

311

312

versus the corifollitropin alfa with a GnRH-antagonist cycle. J Turk Ger Gynecol Assoc 17(3):

155-158 DOI: $10.5152 /$ jtgga.2016.16015.

Liao C, Huang R, Scherer RW, Liang XY. 2015. Prognostic factors associated with clinical pregnancy in in vitro fertilization using pituitary down-regulation with depot and daily low-dose luteal phase gonadotropin releasing hormone agonists: A single center's experience. $J$ Hum Reprod Sci 8(1):30-36.

Londra L, Moreau C, Strobino D, Bhasin A, Zhao Y. 2016. Is the type of gonadotropinreleasing hormone suppression protocol for ovarian hyperstimulation associated with ectopic pregnancy in fresh autologous cycles for in vitro fertilization? Fertil Steril 106(3):666-672 DOI: 10.1016/j.fertnstert.2016.05.019.

\section{Loumaye E, Vankrieken L, Depreester S, Psalti I, de Cooman S,Thomas K. 1989. Hormonal}

changes induced by short-term administration of gonadotropin-releasing hormone agonist during ovarian hyperstimulation for in vitro fertilization and their consequences for embryo

development. Fertil Steril 51(1):105-111. 
323 Mao GH, Feng Z, He Y, Huang YR. 2014. Comparisons of the effects of long-acting and

324 short-acting GnRH agonists on embryo quality, endometrial thickness and pregnancy rate in

325 human in vitro fertilization. Arch Med Sci 10(1):161-166 DOI: 10.5114/aoms.2014.40743.

326 Martínez F, Clua E, Devesa M, Rodríguez I, Arroyo G, González C, Solé M, Tur

R, Coroleu B, Barri PN. 2014. Comparison of starting ovarian stimulation on day 2 versus day

15 of the menstrual cycle in the same oocyte donor and pregnancy rates among the corresponding recipients of vitrified oocytes. Fertil Steril 102(5): 1307-1311 DOI: 10.1016/j.fertnstert. 2014.07.741.

McDonnell R, Marjoribanks J, Hart RJ. 2014. Ovarian cyst aspiration prior to in vitro

Metallinou C, Köster F, Diedrich K, Nikolettos N, Asimakopoulos B. 2012. The effects of

luteinized cells. In Vivo 26(5):835-839. 
339

340

341

342

343

344

345

346

347

348

349

350

351

352

353

354

355

ovarian stimulation in IVF/ICSI cycles. Cochrane Database Syst Rev 5:CD005070 DOI:

10.1002/14651858.CD005070.pub3.

\section{Pereira N, Amrane S, Hobeika E, Lekovich JP, Chung PH, Rosenwaks Z. 2016.}

Cyst aspiration or GnRH antagonist administration for ovarian cysts detected at the start of fresh in vitro fertilization cycles. Gynecol Endocrinol 32(7):562-565 DOI: 10.3109/09513590. 2016.1139565.

Qublan HS, Amarin Z, Tahat YA, Smadi AZ, Kilani M. 2006. Ovarian cyst formation following GnRH agonist administration in IVF cycles: incidence and impact. Hum Reprod 21(3): 640-644.

\section{Razi MH, Mohseni F, Dehghani Firouzabadi R, Janati S, Yari N, Etebary S. 2014. Results}

from adding recombinant LH for assisted reproductive technology treatment: A randomized control trial. Iran J Reprod Med 12(2):111-116.

Ren Y, Yang S, Yang R, Li R, Chen XN, Wang HY, Ma CH, Liu P, Qiao J. 2013. Comparison

of gonadotropin releasing hormone agonist long protocol and gonadotropin releasing hormone antagonist protocol in infertile women. Beijing Da Xuе Xuе Bao Yi Xuе Ban 45(6):877-881.

Ren J, Sha A, Han D, Li P, Geng J, Ma C. 2014. Does prolonged pituitary down-regulation 
356 with gonadotropin-releasing hormone agonist improve the live-birth rate in in vitro fertilization

357 treatment? Fertil Steril 102(1):75-81 DOI: 10.1016/j. fertnstert.2014.03.030.

358 Shahrokh Tehraninejad E, Farshbaf Taghinejad M, Hossein Rashidi B, Haghollahi F. 2017.

359 Controlled ovarian stimulation with r-FSH plus r-LH vs. HMG plus r-FSH in patients candidate

360 for IVF/ICSI cycles: An RCT. Int J Reprod Biomed (Yazd) 15(7):435-440.

361 Siristatidis CS, Gibreel A, Basios G, Maheshwari A, Bhattacharya S. 2015.

362 Gonadotrophin-releasing hormone agonist protocols for pituitary suppression in assisted

363 reproduction. Cochrane Database Syst Rev 9(11): CD006919 DOI: 10.1002/14651858.

364 CD006919.pub4.

Tur-Kaspa I \& Fauser BCJM. 2013. Results: The use of GnRH agonist in IVF protocols.

Available at https://ivf-worldwide.com/survey/the-use-of-gnrh-agonist-in-ivf-protocols/

results-the-use-of-gnrh-agonist-in-ivf-protocols.html.

Xiao JS, Su CM, Zeng XT. 2014. Comparisons of GnRH antagonist versus GnRH agonist

protocol in supposed normal ovarian responders undergoing IVF: a systematic review and 
Figure 1 (on next page)

Flow chart describing drop out of enrolled patients 


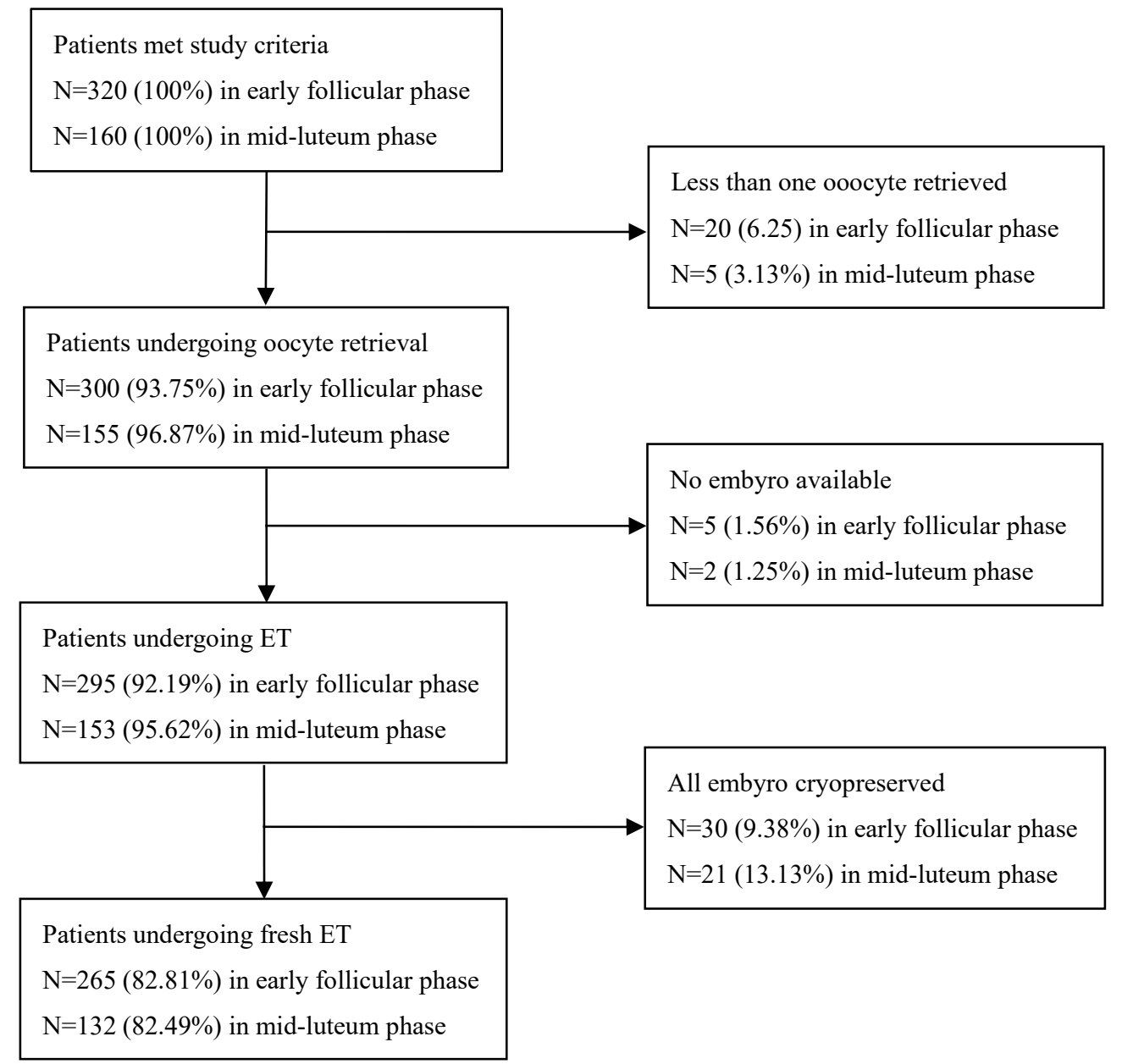


Figure 2 (on next page)

Flow chart of $\mathrm{GnRH}$ agonist prolonged regimen in group 1 


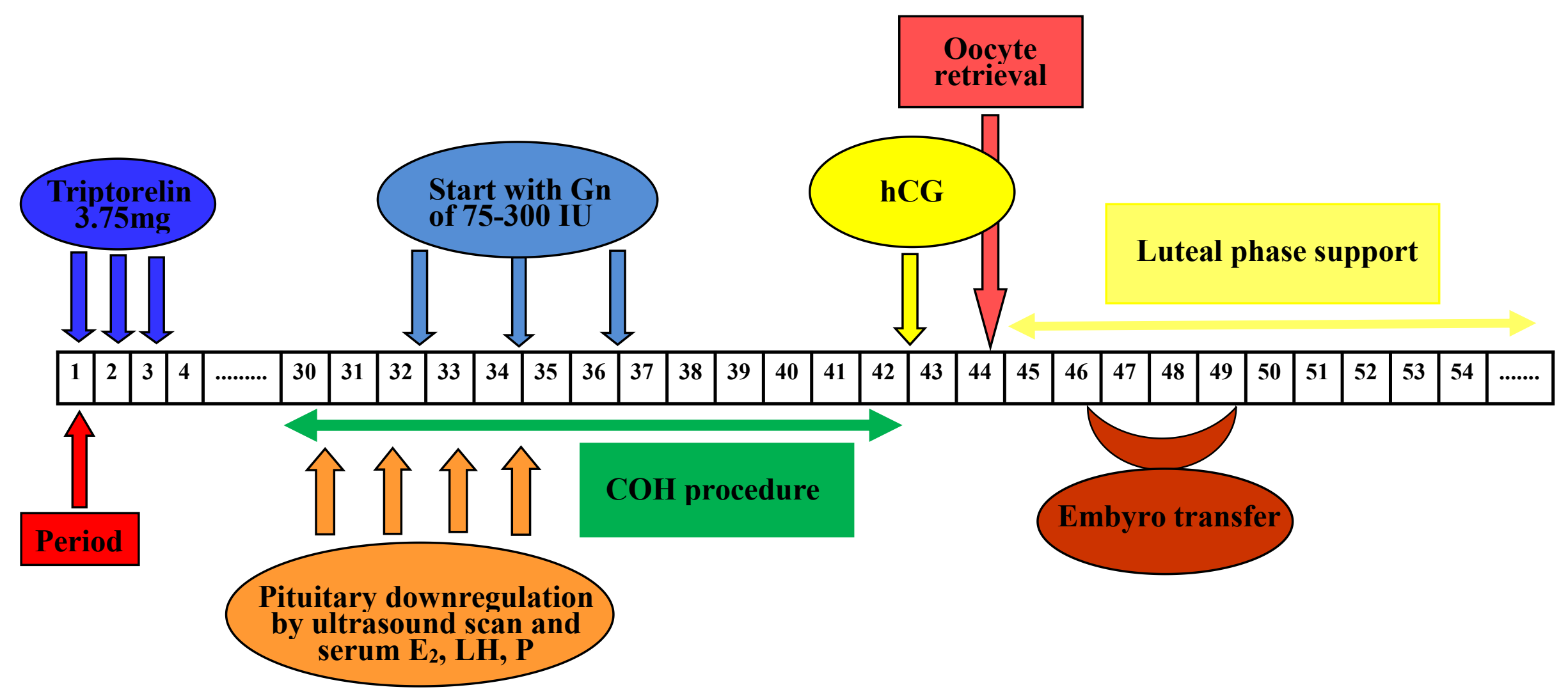


Figure 3 (on next page)

Flow chart of $\mathrm{GnRH}$ agonist prolonged regimen in group 2 


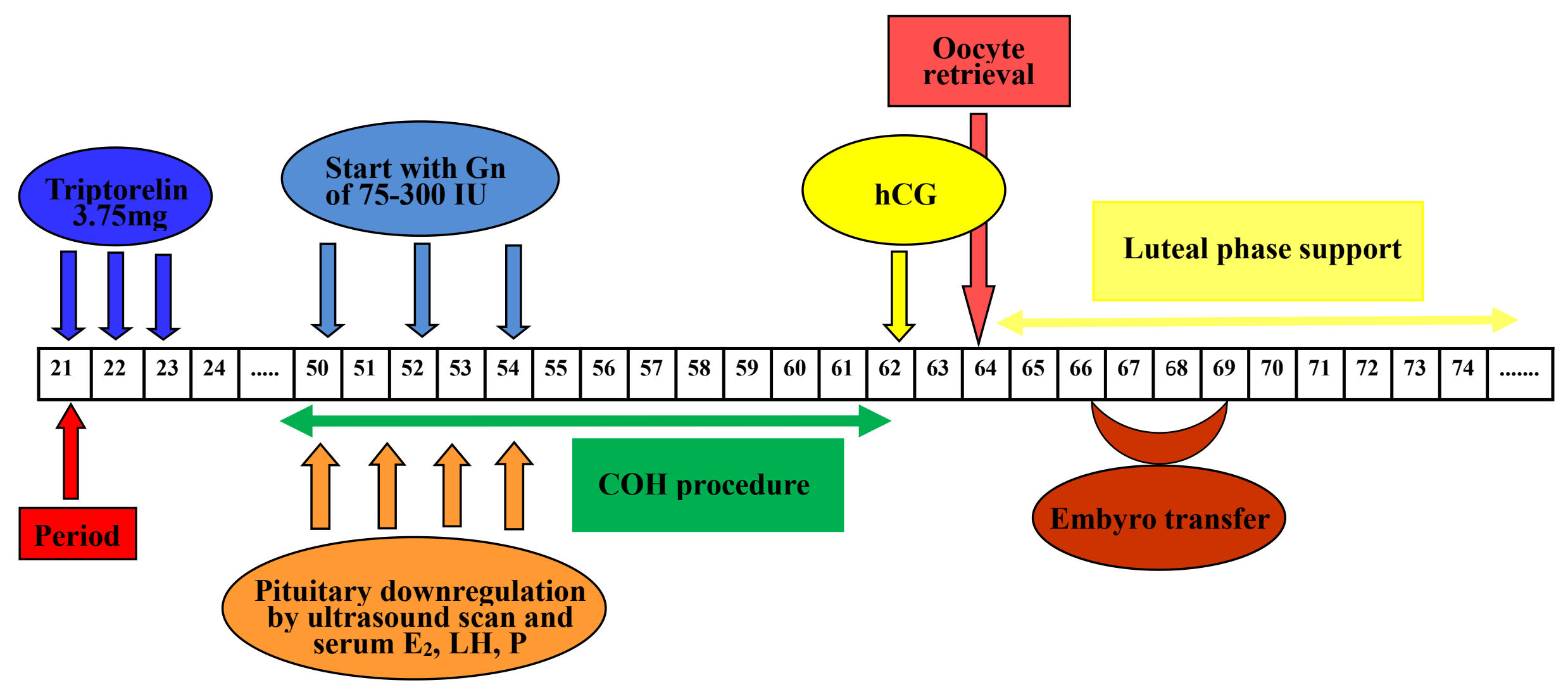




\section{Table $\mathbf{1}$ (on next page)}

Patients' basic characteristics and stimulation variables of the two groups 
Table 1 Patients' basic characteristics and stimulation variables of the two groups

\begin{tabular}{|c|c|c|c|}
\hline & Group $1(n=265)$ & Group $2(n=132)$ & $P$ value \\
\hline \multicolumn{4}{|l|}{ Patient chacteristics } \\
\hline Classifications of infertility & & & 0.915 \\
\hline Primary infertility \%(n) & $44.15(117 / 265)$ & $43.18(57 / 132)$ & 0.855 \\
\hline Secondary nfertility \%(n) & $55.85(148 / 265)$ & $56.82(75 / 132)$ & 0.855 \\
\hline Age (years) & $30.58 \pm 3.57$ & $31.14 \pm 4.1858$ & 0.134 \\
\hline $\mathrm{BMI}\left(\mathrm{Kg} / \mathrm{m}^{2}\right)$ & $21.28 \pm 2.56$ & $21.79 \pm 2.65$ & 0.063 \\
\hline Infertility durations (years) & $3.58 \pm 2.44$ & $3.80 \pm 2.62$ & 0.998 \\
\hline \multicolumn{4}{|l|}{ IVF testifies } \\
\hline Female infertility $\%(n)$ & $48.68(129 / 265)$ & $43.94(58 / 132)$ & 0.373 \\
\hline $\begin{array}{l}\text { Polycystic ovary syndrome } \\
\%(\mathrm{n})\end{array}$ & $8.30(22 / 265)$ & $3.03(4 / 132)$ & 0.052 \\
\hline Endometriosis \%(n) & $10.18(27 / 265)$ & $4.55(6 / 132)$ & 0.056 \\
\hline Male infertility $\%(n)$ & $16.98(45 / 265)$ & $14.39(19 / 132)$ & 0.509 \\
\hline Couple reasons \%(n) & $28.67(76 / 265)$ & $39.39(42 / 132)$ & 0.519 \\
\hline Unkown reasons $\%(n)$ & $5.67(15 / 265)$ & $2.28(3 / 132)$ & 0.126 \\
\hline \multicolumn{4}{|l|}{ Basic homones on the day 3} \\
\hline D3 LH (IU/L) & $4.24 \pm 2.48$ & $4.18 \pm 1.92$ & 0.373 \\
\hline D3 FSH (IU/L) & $5.90 \pm 1.68$ & $6.22 \pm 1.78$ & 0.085 \\
\hline $\mathrm{D} 3 \mathrm{E}_{2}(\mathrm{pg} / \mathrm{ml})$ & $44.56 \pm 14.12$ & $45.80 \pm 16.01$ & 0.432 \\
\hline
\end{tabular}

On the commencing day

Peer) reviewing PDF | (2018:06:29107:3:0:NEW 10 Mar 2019)

LH level (IU/L) $\quad 0.49 \pm .0 .24$

$0.46 \pm 0.33 \quad 0.082$ 
$\mathrm{E}_{2}$ level (pg/ml)

$\mathrm{P}_{4}$ level (ng/ml)

Antral follicular count (n)
$29.26 \pm .12 .19$

$0.35 \pm .0 .17$

$14.68 \pm 5.57$

\section{On the day of hCG}

LH level (IU/L)

$\mathrm{E}_{2}$ level (pg/ml)

$\mathrm{P}_{4}$ level (ng/ml)

Endometrail thickness (mm)

Initial dose of FSH (IU/day)

Days of stimulation (days)

Total dose of FSH (IU)
$0.63 \pm 0.43$

$1994.03 \pm 1220.48$

$0.54 \pm 0.32$

$11.37 \pm 2.03$

$166.09 \pm 53.81$

$11.51 \pm 3.55$

$2243.97 \pm 1070.13$
$26.90 \pm 9.91$

0.121

$0.42 \pm 0.22$

0.373

$12.61 \pm 5.64$

0.001 
Table 2 (on next page)

Laboratory and clinical outcomes of the two groups 
Table 2 Laboratory and clinical outcomes of the two groups

\begin{tabular}{|c|c|c|c|}
\hline & Group $1(n=265)$ & Group $2(n=132)$ & $P$ value \\
\hline \multicolumn{4}{|l|}{ Laboratory outcomes } \\
\hline IVF \%(n) & $64.15(170 / 265)$ & $70.45(93 / 132)$ & 0.211 \\
\hline ICSI \%(n) & $27.17(72 / 265)$ & $18.18(24 / 132)$ & 0.062 \\
\hline IVF/ICSI \%(n) & $8.68(23 / 265)$ & $11.36(15 / 132)$ & 0.392 \\
\hline No. of oocytes retrieved & $11.60 \pm 5.49$ & $12.20 \pm 4.91$ & 0.288 \\
\hline No. of 2 PNs per cycle & $7.98 \pm 4.31$ & $8.02 \pm 3.87$ & 0.946 \\
\hline No. of cleavage per cycle & $7.77 \pm 4.28$ & $7.58 \pm 3.78$ & 0.678 \\
\hline No. of good quality embryos per cycle & $4.19 \pm 3.20$ & $4.08 \pm 3.05$ & 0.745 \\
\hline Rate of fertilized eggs \%(n) & $68.81(2116 / 3075)$ & $65.67(1058 / 1611)$ & 0.030 \\
\hline Rate of cleavage $\%(n)$ & $97.26(2058 / 2116)$ & $94.61(1001 / 1058)$ & $<0.001$ \\
\hline Rate of good quality D3 embyros \%(n) & $50.73(1111 / 2190)$ & $50.99(539 / 1057)$ & 0.911 \\
\hline \multicolumn{4}{|l|}{ Clinical outcomes } \\
\hline No. Embryos transfer & $2.03 \pm 0.34$ & $1.95 \pm 0.31$ & 0.904 \\
\hline D2-D3 \% (mean days) & $73.20(2.97 \pm 0.16)$ & $63.42(2.96 \pm 0.21)$ & 0.091 \\
\hline D4-D5 \% (mean days) & $26.80(4.35 \pm 0.48)$ & $36.58(4.40 \pm 0.50)$ & 0.383 \\
\hline Clinical pregnancy rate $\%(\mathrm{n})$ & $60.38(160 / 265)$ & $61.36(81 / 132)$ & 0.913 \\
\hline Ongoing pregnancy rate $\%(n)$ & $57.74(153 / 265)$ & $57.58(76 / 132)$ & 1.000 \\
\hline Live birth rate $\%(n)$ & $56.23(149 / 265)$ & $57.58(76 / 132)$ & 0.830 \\
\hline Early abortion rate $\%(n)$ & $2.64(7 / 265)$ & $3.79(5 / 132)$ & 0.543 \\
\hline Implantation rate $\%(n)$ & $41.15(221 / 537)$ & $45.91(118 / 257)$ & 0.220 \\
\hline
\end{tabular}


Ovarian cyst formation rate $\%(n)$

Rate of severe early OHSS $\%(n)$

Rate of $E_{2}>4000(\mathrm{pg} / \mathrm{ml})$ on the day of $\mathrm{hCG} \%(\mathrm{n})$

Rate of all embyro cryopreserved \%(n)
$6.42(17 / 265)$

$1.89(5 / 265)$

$6.04(16 / 265)$

$10.17(30 / 295)$
$0(0 / 132)$

0.003

$2.27(3 / 132)$

0.797

$7.56(10 / 132)$

0.560

$13.73(21 / 153)$

0.664

2 


\section{Table 3(on next page)}

Data comparison between cyst and non-cystic subgroups in group 1 
Table 3 Data comparison between cyst and non-cystic subgroups in Group 1

\begin{tabular}{|c|c|c|c|}
\hline & $\begin{array}{c}\text { Cyst subgroup } \\
(n=17)\end{array}$ & $\begin{array}{l}\text { Non-cyst subgroup } \\
\qquad(\mathrm{n}=\mathbf{2 4 8})\end{array}$ & $P$ value \\
\hline \multicolumn{4}{|l|}{ Basic situation } \\
\hline Age (years) & $31.39 \pm 2.98$ & $30.53 \pm 3.61$ & 0.333 \\
\hline BMI $\left(\mathrm{Kg} / \mathrm{m}^{2}\right)$ & $20.92 \pm 2.24$ & $21.31 \pm 2.59$ & 0.351 \\
\hline Infertility durations (years) & $4.18 \pm 3.34$ & $3.54 \pm 2.37$ & 0.303 \\
\hline \multicolumn{4}{|l|}{ Basic homones on cycle day 3} \\
\hline D3 LH (IU/L) & $5.17 \pm 2.97$ & $4.18 \pm 2.44$ & 0.109 \\
\hline D3 FSH (IU/L) & $6.28 \pm 1.77$ & $5.87 \pm 1.68$ & 0.336 \\
\hline $\mathrm{D} 3 \mathrm{E}_{2}(\mathrm{pg} / \mathrm{ml})$ & $49.58 \pm 15.67$ & $44.22 \pm 13.95$ & 0.130 \\
\hline \multicolumn{4}{|l|}{ On the commencing day } \\
\hline LH level (IU/L) & $0.54 \pm 0.22$ & $0.49 \pm 0.25$ & 0.379 \\
\hline $\mathrm{E}_{2}$ level $(\mathrm{pg} / \mathrm{ml})$ & $28.71 \pm 11.20$ & $29.30 \pm 12.28$ & 0.847 \\
\hline $\mathrm{P}_{4}$ level (ng/ml) & $0.33 \pm 0.15$ & $0.36 \pm 0.18$ & 0.624 \\
\hline Antral follicular count (n) & $12.88 \pm 5.49$ & $15.01 \pm 5.56$ & 0.128 \\
\hline \multicolumn{4}{|l|}{ On the day of hCG } \\
\hline LH level (IU/L) & $0.54 \pm 0.27$ & $0.64 \pm 0.44$ & 0.343 \\
\hline $\mathrm{E}_{2}$ level (pg/ml) & $1609.00 \pm 1276.31$ & $2020.42 \pm 1214.77$ & 0.179 \\
\hline $\mathrm{P}_{4}$ level $(\mathrm{ng} / \mathrm{ml})$ & $0.58 \pm 0.41$ & $0.54 \pm 0.31$ & 0.555 \\
\hline Endometrail thickness (mm) & $11.45 \pm 2.49$ & $11.37 \pm 2.00$ & 0.875 \\
\hline Initial dose of FSH (IU/day) & $183.82 \pm 54.44$ & $164.93 \pm 53.59$ & 0.161 \\
\hline Days of stimulation (days) & $11.88 \pm 1.54$ & $11.48 \pm 3.65$ & 0.652 \\
\hline Total dose of FSH (IU) & $2585.29 \pm 950.25$ & $2220.57 \pm 1075.61$ & 0.174 \\
\hline \multicolumn{4}{|l|}{ Laboratory data } \\
\hline No. of oocytes retrieved & $11.00 \pm 4.89$ & $11.65 \pm 5.53$ & 0.640 \\
\hline No. of 2 PNs per cycle & $7.41 \pm 4.42$ & $8.02 \pm 4.31$ & 0.572 \\
\hline No. of cleavage per cycle & $7.35 \pm 4.33$ & $7.78 \pm 4.28$ & 0.682 \\
\hline No. of good quality embryos per cycle & $4.41 \pm 3.34$ & $4.18 \pm 3.20$ & 0.771 \\
\hline Rate of good quality embyros \%(n) & $53.96(75 / 139)$ & $50.51(1036 / 2051)$ & 0.483 \\
\hline \multicolumn{4}{|l|}{ Clinical outcomes } \\
\hline Clinical pregnancy rate $\%(n)$ & $82.35(14 / 17)$ & $58.87(146 / 248)$ & 0.072 \\
\hline Ongoing pregnancy rate \%(n) & $76.47(13 / 17)$ & $56.45(140 / 248)$ & 0.131 \\
\hline Live birth rate $\%(n)$ & $76.47(13 / 17)$ & $54.84(136 / 248)$ & 0.082 \\
\hline
\end{tabular}




\begin{tabular}{llll} 
Early abortion rate $\%(\mathrm{n})$ & $5.88(1 / 17)$ & $2.42(6 / 248)$ & 0.375 \\
Rate of E2 $>4000(\mathrm{pg} / \mathrm{ml})$ on the day & $5.88(1 / 17)$ & $6.05(15 / 248)$ & 0.978 \\
of hCG $\%(\mathrm{n})$ & & & \\
\hline
\end{tabular}

2 